Journal of International Students I 104

Peer-Reviewed Article

ISSN: 2162-3104 Print/ ISSN: 2166-3750 Online

Volume 5, Issue 2 (2015), pp. 104-120

(C) Journal of International Students

http://jistudents.org/

\title{
The Factors That Influence Dietary Habits Among International Students in the United States
}

\author{
Amir A. Alakaam, $M S$ \\ Diana C. Castellanos, PhD \\ Jessica Bodzio, MS \\ Lee Harrison, PhD \\ Marywood University (USA)
}

\begin{abstract}
This study examines the dietary intake changes and factors related to dietary acculturation in international students attending an urban university in the United States. The researchers administered seven focus groups of college-age international students $(n=32)$ between June and August 2012. The participants were enrolled in Northeastern and Midwestern U.S. universities. A qualitative research inquiry was used for data collection, presentation, and analysis. An interview guide was developed to explore the dietary habits of international students. The results show the participants face many dietary challenges as a result of adapting to American culture. The major dietary-related influences include: the food environment, campus environment, religion, and individual preferences. Additionally, the consequences of dietary changes were associated with undesirable health outcomes including weight gain, increased blood glucose levels, increased cholesterol levels, high blood pressure, and mental problems.
\end{abstract}

Keywords: Dietary habits, dietary acculturation, international students, traditional food, Western food.

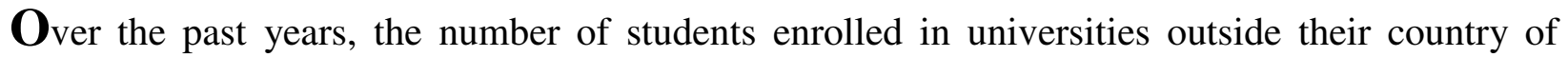
citizenship has risen significantly (Organization for Economic Cooperation and Development [OECD], 2013). In 2011, there were over 4.3 million international students studying worldwide (OECD, 2013). International students attending universities in the United States accounted for the highest percentage (17\%) of international students all over the world (OESD, 2013). The United States is likely to remain one of the most attractive countries and the top host nation in an increasingly competitive market for international students in the foreseeable future (OECD, 2013); therefore, it is important to improve the student experience in the United States (OECD, 2013; Verbik \& Lasanowski - Hobsons, 2007). The purpose of this study is to explore dietary

Summer 2015

http://jistudents.org

Volume $5 \bullet$ Issue 2 
intake changes and factors related to dietary acculturation in the U.S. college-age international student population.

\section{Literature Review}

As international students assimilate to customs and culture of the host country, they also change their dietary practices (Edwards, Hartwell, \& Brown, 2010). Adaption to the host culture is associated with poor diet quality (Brittin \& Obeidat, 2011), primary effects on food choice, eating habits, and physical health (Papadaki, Hondros, Scott, \& Kapsokefalou, 2007; Winham, 2009). Past studies have indicated that changes in individuals' eating habits after they move to the United States can result in undesirable health outcome such as excessive weight gain and development of chronic diseases (Satia-Abouta, Patterson, Neuhouser, \& Elder, 2002; Winham, 2009).

Traditional food is a food with particular characteristic in term of the use of raw ingredients which differentiate it from other processed and convenience food (Trichopoulou, Soukara, \& Vasilopoulou, 2007). Outside of the United States, traditional foods such as bulgur salad in the traditional Mediterranean diet are typically high in fiber, low in saturated and transfat, high in carbohydrates, and frequently have a variety of fresh vegetables and fruits (Ayala, Baquero, \& Klinger, 2008; Chatterjee, 2005; Renzaho \& Burns, 2006; Trichopoulou, Soukara, \& Vasilopoulou, 2007). However, diets in the United States are high in calories from sweeteners and saturated fat, high in salt, and low in carbohydrates and fiber such as fast food (Drewnowski \& Popkin, 1997; Patil, Handley, \& Nahayo, 2009; Winham, 2009). Acculturation is also positively related to length of residence in the United States and with consumption of American food products such as pizza, hotdogs, and American coffee (Brittin \& Obeidat, 2011).

Knowledge of food choices and preferences as well as the factors that influence eating habits of international students from various ethnic groups is necessary in order to provide effective nutrition education and care to an increasingly diverse population (Brittin \& Obeidat, 2011; Verbik \& Lasanowski - Hobsons, 2007). However, little is known about college-age international students' food experience and dietary habits after arrival in the United States. To gain understanding of dietary behaviors among international students, the following question was investigated: What factors influence dietary acculturation among a group of college-age international students in the United States.

\section{Research Method}

In this study, the grounded theory was used for data collection, analysis, and presentation. The grounded theory of qualitative research was utilized to explore dietary acculturation in the target population. Grounded theory research is a qualitative tradition built on compared concepts (Glaser, 1992; Strauss \& Corbin, 1990) and is viewed as a relatively easy approach to qualitative research (Bryant \& Charmaz, 2007), that enables theories to emerge from the data collected (Urquhart, 2001). Grounded theory allows processes to emerge and discover the relevance of the data; the theory obtains an abstract analytical outline of phenomena that related to a specific situation (Creswell, 1998). This method follows a systematic yet flexible process to collect, code, and make connections among the finding; and develop theories that emerge from the data (Hage, 1972). 


\section{Researchers and Trustworthiness}

In the qualitative study, the researchers consider as an instrument for data collection and analyses (Bodgan \& Biklen, 2007). In the current study, the researchers have a different background. The first author is an international master students, had participated in previous research related to international student dietary habits and nutrition policy. The second and third authors are faculty members in the Nutrition departments in Marywood University in Pennsylvania and registered dietitians (RD). The fourth author is a full professor, a chairperson of Nutrition and Dietetics Department in Marywood University and an RD. The authors had several years' experiences in the qualitative and quantitative research related to dietary acculturation, dietary habits of immigrants, and food researches. The researchers make effort to prevent the personal connections to international students affects the study process and the data analyses. The researchers endeavored to make the data more rigorous and far from biases by using multiple researchers in the coding process and data analyses.

To ensure trustworthiness a team was recruited to provide feedback on the article's manuscript and to involve the authors in questioning data collection and analyses. This team included two faculty members in the nutrition department and three doctoral students from two different U.S. Universities who study dietary acculturation, nutrition policy, and food system. Feedback from other researchers and student informants was used to support data finding and analyses, and to confirm content validity. The data assumptions about international students' dietary habits were honestly reported and discussed among authors to ensure data quality and trustworthiness, as these method used to inform dependability, conformability, and credibility (Lincoln \& Guba, 1986).

\section{Procedure}

The Institutional Review Board approved this study under Marywood University Institutional Review Board. The primary researcher conducted seven focus group interviews using an interview guide. There were four to six participants in each focus group. The interviews were held in a location referred by the participants as public and easily accessible, the location identified after the researcher asked all the participants where they would like to meet. During the focus groups, each participant first read and signed an informed consent form before participating. The primary researcher administered the interview guide (described below) to each focus group and utilized a note taker. Probes were used to further explore or clarify statements made by the participants. Each focus group was audio-recorded and then transcribed verbatim by the trained research assistant within 48 hours after the interview was completed. The focus group recruitment and process continued until data saturation was reached.

\section{Focus group interview guide}

The principal investigator (PI) developed a focus group interview guide. The questions in the guide were based on literature review and used concepts from previous studies (Brittin \& Obeidat, 2011; Edwards, Hartwell, \& Brown, 2011; Jabber, Brown, Hammad, Zhu, \& Herman, 2003; Pan, Dixon, Himburg, \& Huffman, 1999; Yeh et al., 2008). The guide generated discussion around the 
following constructs: (a) previous dietary habits prior to coming to the United States, (b) current dietary habits and food choice changes, (c) the influence of different factors on these changes, and (d) the overall health effects after living in United States. Appendix 1 provides list of the questions in the guide. The guide was reviewed by three registered dietitians. Furthermore, it was pretested with three international students (one undergraduate student and tow graduate students) representing the target population for clarity and understanding, and to confirm content validity. Changes were made based on the review and pretest.

Table 1

Focus Group Participant Demographic and Social Characteristics $(\mathbf{n}=32)$

\begin{tabular}{lll}
\hline Characteristic & Category & Number of participants \\
\hline Sex & Male & 20 \\
Home country & Female & 12 \\
& Asia & 15 \\
& South Asia & 3 \\
& Middle East & 13 \\
Race & Latin American & 1 \\
& White & 13 \\
Time in United States & Latino & 1 \\
& Asian & 18 \\
Status & 6 months - 2 years & 9 \\
\multirow{3}{*}{ Residence in the United States } & 2-4 years & 23 \\
& Single & 18 \\
Religion & Married & 14 \\
& Northeast & 17 \\
& Midwest & 15 \\
& Muslim & 17 \\
& Christian & 7 \\
& Buddhism & 3 \\
Level of education & Sikhism & 1 \\
& Confucius & 1 \\
& None & 3 \\
& Undergraduate & 8 \\
& Graduate & 24 \\
\hline
\end{tabular}

\section{Participants}

Snowball sampling was used to recruit participants. This type of sampling is useful and cost efficient when further participants of a specific topic are needed and a limited number are available (Salganik \& Heckathorn, 2004). The primary researcher contacted acquaintances that were college students at five different U.S. urban universities; three in the Northeastern and two in the Midwestern United States. The acquaintances provided fellow international students with the necessary information regarding the study. If the international student expressed interest in participating, then he or she contacted the primary researcher in order to acquire additional information. The inclusion criteria for participation were as follows: aged 18-40 years, fluent in the English language, had been in the United States between six months to four years, and 
enrolled in a U.S. university. All participants were international students. This included holders of U.S. student visas (F-1 Visa, J-1 Visa, and M-1Visa), as well as individuals who were born in the United States but left before the age of two and only returned upon entrance into a university.

There were seven focus groups with a total of 32 male and female international students, of the 32 students, 20 were male and 12 were female. Age ranges for the participants from 19 to 38 years. Participants represented five U.S. urban universities; three were located in the Northeast and two in the Midwest. Table 1 provides characteristics information of the participants.

\section{Analysis}

The analysis started after the first interview. Three trained coders in qualitative inquiry analyzed the data to check for consistency, two were nutrition graduate students and one was nutrition faculty in Marywood University. The grounded theory of qualitative research was used for data collection, analysis, and presentation (Urquhart, 2001). Grounded theory research is a qualitative tradition built on compared concepts (Glaser, 1992; Strauss \& Corbin, 1990) and is viewed as a relatively easy approach to qualitative research (Bryant \& Charmaz, 2007), that enables theories to emerge from the data collected.

The researchers followed this systematic method to collect and code the data; and to develop theories from the data collected during the interviews (Hage, 1972). The researcher analyzed the data collected from participants by using constant comparison method as one of component included in grounded theory to search for recurring themes in the transcripts (Bryant \& Charmaz, 2007). During data collection and analysis the researcher grouped and conceptually labeled the similar data during a process called open coding, which is the first stage to analyze the data in the qualitative research (Urquhart, 2001). The open coding yielded several ideas which became the key findings of the data analysis (Strauss, 1987; Urquhart, 2001). Then, the researcher categorized, linked, and organized concepts by relationship in a process called axial coding. Conditions and dimensions were developed, and finally through an interpretive process called selective coding, the study's themes emerged (Glaser, 1978; Glaser, 1967; Strauss, 1987). In this stage, the resulting strategies were fully shaped out and the core category became saturated (Urquhart, 2001). The researchers used the participants own language to identify the categories and to emerge the themes and to represent the results.

\section{Results}

Analysis of data extracted four major themes associated with dietary acculturation among international students in the United States: (1) eating patterns, (2) influences on dietary habits changes after migration, (3) resistance to changes in dietary patterns, and (d) the consequences of these changes.

\section{Themes}

Eating Patterns. According to this study, eating patterns were defined as the decisions that an individual makes when choosing which foods to eat. These patterns were divided into two subcategories: (a) eating patterns in the home country and (b) eating patterns in the United 
States. Table 2 provides the study findings about participants dietary habits in the home countries versus the United States.

Table 2

Dietary Habits in the Home Countries vs. Dietary Habits in the United States

\begin{tabular}{lll}
\hline Dietary habits & Home countries & United States \\
\hline Eating patterns & Mainly traditional food & Mainly American food \\
& Simple and basic & Convenience food \\
Commensal eating & Eating alone most of the time \\
Meals & Some cooked meal & Unstructured meal \\
& Specific meal time & Consuming more meals \\
& Eating meals with three courses & Large food portion sizes \\
& Small food portion size & Skipping breakfast / light breakfast \\
& Consuming breakfast daily & Late night meal available \\
& No late night meal available. & Less varied \\
& More varied & More fast food and meat \\
& Less meat & Less fresh fruits and vegetables \\
& Fresh fruits and vegetables & Consuming snacking and desserts. \\
Limited snacking and desserts & Drinking traditional tea & Drinking coffee \\
\hline
\end{tabular}

A. Eating patterns in the home country. The main foods that the participants consumed in the home country were traditional foods, which were defined as the foods that the participant normally ate and were culturally familiar. These foods were fresh prepared, simple, and basic, such as meat, eggs, fish, beans, vegetables, fruit, seeds, and milk. (Drewnowski \& Popkin, 1997; Winham, 2009).

Participants consumed a variety of traditional foods such as: eggs, bread, rice, meat, fruits, and vegetables. The participants indicated that the foods consumed in the home countries were fresh with limited frozen and processed products. Furthermore, participants mentioned that there were not any food labels in the home countries. They tended to associate food labeling with processed foods, and therefore believed that the foods they formerly consumed were fresh and healthy. The participants mentioned that fast food restaurants in their home countries were expensive and the units offered traditional food items (like rice in Indonesia).

When asked about meals, most of the students (80\%) said that meals are home cooked, occur mostly at homes on a daily basis, are varied, filling, and small in portion size, and take place at a specific time during the day. Breakfast is around 8:00 a.m., lunch is around 1:00 p.m., and dinner is around 7:00 p.m., with limited desserts and snacking, and no late night meal. Most of the participants drank traditional tea (90\%) (black or green tea leaves brewed and boiled in water for several minutes) the majority of the time.

Usually the woman of the household, such as a mother or wife, provided and prepared the meal. One male student from Pakistan summed up these patterns:

Back home we ate all sorts of foods. We have all sorts of cuisines over there; I've eaten all types of foods from my home country. I ate with my family because I lived with 
them. Friends, at least I had one meal with them, maybe lunch, and then I met with my family for dinner

Another student from Iraq said:

A homemade meal, rice is the major dish, bread of course in the breakfast, lunch, and dinner. For breakfast I ate eggs and drank tea and I never drank coffee. I ate bean or fresh okra stew with rice for lunch.

B. Eating patterns in the United States. The main foods the participants eat in the United States were associated with an American diet; according to this study, defined as: foods that are not native to the individual home country and are commonly high in fat, meats, and sugar; and low in fresh fruits and vegetables; however, this food chose by participants and cannot represent all American foods.

Students who moved to the United States indicated a higher consumption of fried food, meats, sugar, salt, convenience food (frozen, cans, and ready to eat food), cereals, bread, dairy products, soda beverages, snacks, desserts, and less fruits and vegetables. The majority of the students mentioned drinking coffee in the United States instead of tea and skipping breakfast or eating a light breakfast. The students agreed that traditional food items are different in the United States than in their home country in terms of price and quality. It is difficult to find the ingredients of traditional food in most of the local stores in the United States. The students said that the traditional food items in the United States are modified or processed (i.e. have too much salt and sugar). An Asian female student said:

I eat more meat here than in China, especially for lunch and dinner. For breakfast I have milk and cereal. I don't drink soy milk anymore, it's not common here. I don't really drink tea as often and sometimes I'll drink coffee. And since I go to school and my schedule is different, my lunch is different every day. Sometimes it may be at 12 o'clock, sometimes 2 o'clock. I might have snacks like energy bar after lunch. I didn't do that in China.

When asked about meals most of the students said meal patterns were unstructured, the students were consuming more meals in the United States (four meals) at different times according to free time and what was available. The portions provided at meals were larger. Most of the students were eating alone or with friends. Some of the students tried to cook but the majority of them were eating convenience foods or in the fast food restaurants due to lack of time and cooking skills. A Muslim student said:

When I came to the States my food changed a lot. For example, I eat cereal with milk and coffee in the breakfast, no more tea. I go to Subway for lunch twice a week or cook in the home some salad and cheese sandwich. I eat alone. I don't consume traditional food because it is not available here near the campus and in most of the regular stores they don't have Halal food. 


\section{Influences on Dietary Habits Changes after Migration}

Students referred to the food environment, individual preferences, religion, time, and campus environment as the major factors that influence the process of dietary habits change and the adaptation to the U.S. culture.

Food environment. This environment is a collection of several factors such as: place where food is obtained, food prices, community characteristics, restaurant proximity, store availability, and other factors that influence individual's food choices and eating habits (Willis \& Buck, 2007).

Subcategories of food environment are food access and food availability.

Food access. Food access, according to this study, was defined as having sufficient resources economically and physically to obtain appropriate foods for a nutritious diet. Food access is also associated with economic factors, time of immigration, and/or employment status (Winham, 2009). Across the focus groups, access to the fast and convenience foods were easy, while traditional food items were difficult to obtain. The factors that play the major role in food access are (a) economics and (b) transportation.

A. Economic influences in the United States. Food cost plays a role in the dietary pattern changes. The participants from Middle East and Asia said that meat and vegetables are in the same price range in the United States, whereas in the home country vegetables are cheaper than meat, so the student prefer to buy meat products in the United States. A student from Malaysia said:"I consume more meat here because it is cheaper compared to fruits and vegetables."

The participants agreed that fast and convenience food is cheaper and this makes them consume more of these items; furthermore, the traditional food items are more expensive and poor in quality. A participant from Turkey said: "The food in Turkish stores in the United States have a passed expiration date or is very near, like a few months, so I can't save it for a long time, and it is expensive."

B. Transportation. The participants mentioned that the traditional stores were located in big cities while convenience food stores and fast food restaurants are within walking distance. Public transportation to the traditional stores is limited, especially in the Northeast universities. The students rely on friends who have cars for trips to the stores, or they go to shop as a group once weekly or monthly. A student in one of the Northeastern universities said: "The first year I didn't have a car so that was a struggle. I would ask my friends to take me or get things for me. The public transportation is bad here."

Food availability. Food availability was defined as: having available quantities of food on a consistent basis near a person's living area (Yusuf et al., 2001). The participants mentioned that there are fewer traditional food ingredients available in the United States, whereas there are more American food choices. In general there is less fresh produce in the United States. A participant from Korea said:

The biggest thing influencing my diet in United States is the system of the grocery in America. In Korea we have many kinds of food stores near home especially small stories 
for fruits and vegetables so we consumed fresh food daily. But in the United States the stores are not close by.

Across the focus groups, food availability was dependent on the area (Northeast vs. Midwest). The traditional food availability was more difficult in the Northeast than in the Midwest because the universities in the Midwest were situated in major cities. The students agreed that traditional food stores were only found in major cities (at two to three hours away). An Asian student in one of the Northeastern universities said:

There is nowhere I can get the Japanese food in Scranton, Pennsylvania. I would drive two or three hours to New Jersey or Philadelphia to get Japanese food. That's the main problem I have in Scranton.

The unavailability of traditional food made some students ask parents to ship the traditional ingredients from the home country every couple of months. A female student from Korea said: "My mother ships the ingredients from Korea to me every three to six months. Even if the shipment is expensive she still does that."

Individual preferences. Every student has unique likes and dislikes concerning food. In general most prefer traditional food, a good quality product, and a variety of produce. Some of the students tried to cook traditional foods while others adapted to the U.S. culture. A male participant from Saudi Arabia said:

I tried to cook by myself, bring the recipes and bring all the spices. I never cooked before. I tried to adapt myself to United States. In the morning it can be cereal or eggs. In Saudi Arabia I never ate cereal.

Religious influences. Muslim students were concerned about consuming Halal food (foods that are allowed under Islamic guidelines). Due to the limited access to the Halal food items in the United States, they are consuming fewer meats, eating less in restaurants and on campus. One of the Muslim participants in Northeastern University said:

I eat less chicken because of my religion. I can't find Halal meat here. I haven't seen any Halal food in the stores. Even in the big stores like Giant or Wal-Mart there are no Halal foods.

Time. Most of the students claimed that busy schedules were one of the factors that made them consume more convenient and ready to eat food. Attending classes, working, and studying, on a daily basis makes the students eat convenience foods instead of cooking or eating healthy foods. One student in one of the Midwest universities said, "There is a store near the dorm, but I don't go there because I don't have time. I must plan ahead to cook and eat healthy on a busy schedule."

Campus environment. The students mentioned that the campus environment and geographical area play major roles on dietary habits changes. The students' dietary patterns changed because of the meal plans (students living in the dorm being forced to have meal plans). 
Several students agreed that the buffet-like style in the cafeteria and having salt and sugar available on tables makes them consume more food on campus, and it has led to weight gain. An Asian student in one of the Northeastern universities said: "They should construct a better healthy meal plan for students. Having salt on the table allows people to keep adding more salt." The students also said that foods are more expensive on campus with less variety , "I'm trying to avoid food on campus because it is very expensive and I'm not sure about the quality."

\section{Resistance to Changes in Dietary Patterns}

There were different factors that led to a resistance to changes in dietary behavior from a traditional diet to more of an American diet. Family structure influenced dietary change. For example, students who have a spouse with them reported fewer changes on dietary habits; while students who have children with them in the United States reported more changes in dietary habits. A female participant from Indonesia said:

I have to study and take care of my kids. I skip my breakfast because I'm busy with my kids and I don't have time. Sometimes I try to cook rice but my children don't really like rice so I will replace it.

Most of the participants see eating at home, cooking, and preparing traditional meals as a way to resist the dietary habits changes and to eat healthier. Some students prepare food when they go to work because it is cheaper and healthier. A participant said:

I prefer to bring my own food that I made to campus because it's healthier, cheaper, and traditional. When you buy chicken in the store it costs three dollars, but if you buy a sandwich it also costs 3 dollars, so if you cook by yourself you will save money that way.

Ethnic restaurants in the United States also play a role in the resistance. The participants consider the ethnic restaurants poor representations of traditional food, and they are more expensive than fast food restaurants. A student from Japan said:

For Japanese food, we don't use much salt or oil. In the United States, they use salt, oil, and sugar; they just put everything. Sometimes when I go to a Japanese restaurant it is owned by different people, not Japanese people. They just use the name of the Japanese restaurant, but how they prepare the food is totally different. That's why I don't eat there.

\section{Consequences of Dietary Pattern Changes}

Participants in all focus groups responded that health consequences and feelings of guilt are the main consequences of dietary habit changes.

Health consequences. The students reported that they are concerned about health. After the move to the United States, the participants reported a variety of health problems, including high blood pressure, increased blood glucose levels, and increased cholesterol levels (as selfreported by participants). A male student from China said: "I had to reduce my amount of sugar in the United States because my sugar level was high and my friend had problems with diabetes after he moved here." 
The majority of the participants reported weight gain in the United States due to eating more meals, eating outside the home, and exercising less because of busy schedules and cold weather in the United States. The students confided that foods products in the United States are large in size and sweeter in general in comparison with home countries. A female student from Iraq said:

When you go to the restaurant in the United States the dish is very big; even the drinks are so big. Beverages in the United States are sweeter than in Iraq. I think that's why I gained weight; because there is a lot of food that is too sweet combined with a lack of exercise. Even if I order ice cream and I say I want one scoop, the scoop is too big.

Female student from Korea said:

The grocery stores here have big products like a gallon of milk. In Korea we only have a quarter gallon. They also have more prepared foods, which have more chemicals, and that affects my body.

The students are trying to modify eating habits to become healthier by choosing restaurants with less fried products like Subway. Some of the participants connected bad taste of food and unhealthy food to inorganic food products and the use of hormones in meat products. Male students from Indonesia said: "I'm gaining weight while I am eating healthy food because of the hormones they used in the food's processing and I do not exercise."

Feelings of guilt. Several participants reported feelings of guilt in the United States due to eating more meals and consuming unhealthy food. Additionally, Muslim students felt mentally ill because of not consuming Halal food. The Halal food is not readily accessible in the United States, especially in the Northeast. A Muslim student said: "The diet will not be just better if there was more Halal, but also it will be better for our mental health as international students."

\section{Discussion}

There are several changes to dietary habits such as: skipping breakfast, eating fewer fruits and vegetables, and convenience food consumption are universal among college-age students and are not specific to international or domestic students (Deshpande, Basil, \& Basil, 2009; Huang, Song, Schemmel, \& Hoerr, 1994). However, this study showed that there are several unique challenges such as: the limitation in the food availability and access, and consequences of dietary habit changes specifically related to international students' dietary habits as a result of adapting to the U.S. culture. In this study, the consequences of dietary changes were associated with undesirable health outcomes including weight gain, increased blood glucose levels, increased cholesterol levels, high blood pressure, and mental problems (as self-reported by participants). There are several factors that may contribute to these changes, including food environment, campus environment, time, religion, and individual preferences.

The information on factors associated with the adaptation of the international populations' diets in the United States is limited. Past research for this population has focused on 
the dietary habits of international students in general (Chatterjee, 2008). This was one of the few studies, to our knowledge, that examine the factors that influence the dietary habits of collegeage international students in the United States from various origins and ethnic groups.

On the basis of the study, college-age international students faced difficulties with dietary habits after moving to the United States Prior studies confirm these findings in terms of international students' dietary changes post-immigration from a diet of traditional food to more Western dietary behaviors (Brittin \& Obeidat, 2011; Devine, Connors, Sobal \& Bisogni, 2003; Edwards, Hartwell, \& Brown, 2011; Pan, Dixon, Himburg, \& Huffman, 1999; Roshania, Narayan, \& Oza-Frank, 2008; Willis \& Nkwocha, 2006; Yeh et al., 2008).

For example, Pan et al. (1999) collected information on changes in the dietary habits among 63 Asian students in the United States who had been in the United States at least three months before the start of the study. The study reported significant increases in the consumption of fats, snack, dairy products, and fast food. However, Pan et al. (1999) reported decrease in the number of meals consumed per day primarily because of the students' class timetables; this was not reported in our population.

Furthermore, several factors have been shown to influence the dietary intake of international students. Participants mentioned that the lack of consumption of traditional food is due to high costs, poor quality, lack of time, and limited access. These finding are consistent with earlier studies that examined the dietary intake changes post immigration (Devine, Connors, Sobal \& Bisogni, 2003; Willis \& buck, 2007; Yeh et al., 2008). Yeh et al. (2008) showed that the high cost of fruit and vegetables and the lack of energy and preparation-time were common barriers to consuming traditional foods among African American, Hispanic, and Caucasian international populations in North Carolina.

Lastly, findings from the sample of college-age international students of this study underscore the possible connection between dietary acculturation and health outcomes in the United States, this was supported by previous research in the United Kingdom, which surveyed 226 international postgraduate students (Edwards, Hartwell, \& Brown, 2010). The findings showed that overall food neophobia increased three months post-immigration. Both Asian and European students reported small changes in eating habits. This study demonstrated that individuals showing neophobia had poor food habits with a possibility of negative health consequences due to their overall changed food consumption patterns (Edwards, Hartwell, \& Brown, 2010).

Other researchers also found evidence of negative health outcomes for international populations in the United States such as excessive weight gain, obesity, food neophobia, and diabetes (Edwards, Hartwell, \& Brown, 2010; Jabber, Brown, Hammad, Zhu, \& Herman, 2003; Mansell, Bennett, Northway, Mead, \& Moseley, 2004; McDonald, \& Kennedy, 2005; Rondinelli et al., 2011; Roshania, Narayan, \& Oza-Frank, 2008). However, the role of dietary acculturation on weight gain and other health measures in international students needs to be further explored.

\section{Limitations}

Several limitations associated with this study should be noted. A qualitative approach was appropriate for this study; due to the limited number of participants from only two geographical areas in the United States, cultural limitation might exist and caution must be taken when generalizing these results onto the overall international student population. However, this study 
could provide a framework of exploring dietary acculturation in other universities' international students. The focus group interview format enhanced data through participant interaction, but may reduce response time of individuals who are less verbal and able to share perspectives (Mansell et al., 2004).

The analysis style in this qualitative study used subjective interpretation to identify the themes; however, three independent coders were used to check for consistency and to improve reliability of findings. One of the limitations that must be considered when analyzing data is the limited ability of the participant to fully express his or her thoughts and opinions by English language. Some of the participants did not speak English as a first language. This is certainly something to consider in the future.

\section{Implications for Future Practice and Research}

Knowledge of food choices and preferences as well as the factors that influence eating habits of international students from various ethnic groups is necessary in order to provide effective nutrition education and care to an increasingly diverse population (Brittin \& Obeidat, 2011). The present findings could have practical implications informing the development of university policies that facilitate a healthy food environment that is culturally appropriate for international students. We believe that implementing new practices would be beneficial for both the university and the student body. The university will attract more international students and thus increase its diversity; not only the international, but also the American students could benefit from these changes. Implementing dietary programs to improve the nutritional health of students is not a unique claim to international students. The health of everyone concerned would most likely improve with implementing specific programs. Therefore, this research suggests that the university work with the food service companies to offer a wider variety of foods and more dietary options for international students such as offering Halal food items to Muslim students. Several U.S. universities have a high percentage of Muslim students, but only a few universities offer Halal food (Islamic Food and Nutrition Council of America [IFANCA], 2011). Students, even freshmen who have no choice but to live in on-campus dormitories, were not offered meals that adhered to their dietary needs (IFANCA, 2011).

It is also advised that the university bring together local farmer's markets with food service companies on campus. Ethnic food stores could be invited to participate as well; it may be beneficial for all parties to implement a system in which each store corresponds with a day of the week. This fixed schedule would help students remember when they could obtain certain traditional foods, and it would also encourage the merchants to devote one day per week to the success of this endeavor. International students would possibly benefit from having a kitchen available in their dormitories. In general, these practices would provide fresher and healthier food choices for everyone.

The university, during new student orientations and special programming throughout the semester, could increase awareness of international and ethnic food stores in addition to American grocery stores. It is important to let students of all backgrounds, especially international, know the array of available food options and be introduced to their new food environment. If not already occurring, shuttle services could be provided that incorporate routes to diverse places, including traditional stores and restaurants. Furthermore, the university could partner with area businesses in order to provide its students with more flexible meal plan spending options in which students could use the university food allotment at local grocers. This 
could lead to increased levels of student satisfaction as students will have more varied dietary options. In turn, the university would support the local communities and economies. Carrying out these recommendations has the potential to offset negative consequences associated with the change in dietary habits of international students post-immigration.

\section{Conclusion}

The international students face many dietary challenges as a result of adapting to the U.S. culture. In this study, the consequences of dietary changes were associated with undesirable health outcomes including weight gain, increased blood glucose levels, and mental problems (as self-reported by participants). There are several factors that may contribute to these changes, including campus environment, individual preferences, and food environment.

Knowledge of food practices and preferences of international students from various ethnic groups is necessary in order to provide effective nutrition education and care to an increasingly diverse population. Our findings highlight the need for university nutrition policies and nutrition service providers to recognize the real nutrition needs of the international students. However, the role of dietary acculturation on weight gain and other health measures in international students needs to be further explored.

\section{References}

Ayala, G., Baquero, B., \& Klinger, S. (2008). A systematic review of the relationship between acculturation and diet among Latinos in the United States: Implications for future research. Journal of the American Dietetic Association, 108(8), 1330-1344. doi:10.1016/j.jada.2008.05.009

Bogdan, R. C., \& Biklen, S. K. (2007). Qualitative research for education: An introduction to theories and methods (5th ed.). Boston, MA: Allyn \& Bacon.

Brittin, H., \& Obeidat, B. (2011). Food practices, changes, preferences and acculturation of Arab students in U.S. universities. International Journal of Consumer Studies, 35(5), 552-559.

Bryant, A., \& Charmaz, K. ( (2007). The sage handbook of grounded theory. London, UK: Sage.

Chatterjee, S. (2008). Health and dietary issues affecting eastern Europeans and Middle easterners. California Food Guide. Retrieved from http://www.dhcs.ca.gov/formsandpubs/publications/Pages/CaliforniaFoodGuide.aspx.

Creswell, J. W. (1998). Qualitative inquiry and research design: Choosing among five traditions. Thousand Oaks, CA: Sage.

Deshpande, S., Basil, M. D., \& Basil, D. Z. (2009). Factors influencing healthy eating habits among college students: An application of the health belief model. Health Marketing Quarterly, 26(2), 145-64.

Devine, C., Connors, M., Sobal, J., \& Bisogni, C. (2003). Sandwiching it in: Spillover of work onto food choices and family roles in low and moderate-income urban households. Social Science \& Medicine, 56(3), 617-630.

Drewnowski, A., \& Popkin ,B. M. (1997). The nutrition transition: New trends in the global diet. Nutrition Reviews, 55(2), 31-43.

Edwards, J., Hartwell, H., \& Brown, L. (2010). Changes in food neophobia and dietary habits of international students. Journal of Human Nutrition and Dietetics, 23(3), 301-311. 
Glaser, B. G., \& Strauss, A. L. (1967). The discovery of grounded theory: Strategies for qualitative research. Chicago, IL: Aldine Publishing Co.

Glaser, B. G. (1992). Emergence vs. forcing: Basics of grounded theory analysis. Mill Valley, CA: Sociology Press.

Glaser, B. G. (1978). Theoretical sensitivity: Advances in the methodology of grounded theory. Mill Valley, CA: The Sociology Press.

Hage, J. (1972). Techniques and problems of theory construction in sociology. New York, NY: Wiley.

Huang, Y., Song, W. O., Schemmel, R. A., \& Hoerr, S. M. (1994). What do college students eat? Food selection and meal pattern. Nutrition Research, 14(8), 1143-1153.

Islamic Food and Nutrition Council of America (IFANCA). (2011). Halal food options in U.S. higher education campus cafeterias. Retrieved from http://www.ifanca.org/

Jabber, L., Brown, M., Hammad, A., Zhu, Q., \& Herman, W. (2003). Lack of acculturation is a risk factor for diabetes in Arab immigrants in the US. Diabetes Care, 26(7), 2010-2014.

Lara, M., Gamboa, C., Kahramanian, M. I., Morales, L. S., \& Bautista, D. H. (2005). Acculturation and Latino health in the United States: A review of the literature and its sociopolitical context. Annual Review of Public Health, 26, 367-97.

Lincoln, Y., \& Guba, E. G. (1986). But is it rigorous? Trustworthiness and authenticity in naturalistic observation. In D. Williams (Ed.), Naturalistic evaluation. New Directions for Program Evaluation (No. 30, pp. 73-84). San Francisco, CA: Jossey-Bass.

Mansell, I., Bennett, G., Northway, R., Mead, D., \& Moseley, L. (2004). The learning curve: The advantages and disadvantages in the use of focus groups as a method of data collection. Nurse Researcher, 11(4), 79-88.

McDonald, J. T., \& Kennedy, S. (2005). Is migration to Canada associated with unhealthy weight gain? Overweight and obesity among Canada's immigrants. Social Science \& Medicine, 61(12), 2469-2481.

Organization for Economic Cooperation and Development (OECD). (2013). Education at a Glance. Retrieved from http://www.oecd.org/edu/eag2013\%20(eng)-FINAL\%2020\%20June\%202013.pdf

Pan, Y. L., Dixon, Z., Himburg, S., \& Huffman, F. (1999). Asian students change their eating patterns after living in the United States. Journal of the American Dietetic Association, 99(1), 54-57.

Papadaki, A., Hondros, G., Scott, J. A., \& Kapsokefalou, M. (2007). Eating habits of university students living at, or away from home in Greece. Appetite, 49(1), 169-176.

Patil, C. L., Handley, C., \& Nahayo, P. D. (2009). Unpacking dietary acculturation among new Americans: Results from formative research with African refugees. Journal of Immigrant and Minority Health, 11(5), 342-358.

Renzaho, A., \& Burns, C. (2006). Post-migration food habits of sub-Saharan African migrants in Victoria: A cross-sectional study. Nutrition \& Dietetics: The Journal of the Dietitians Association of Australia, 63(2), 91-102.

Rondinelli, A., Morris, M., Rodwell, T., Moser, K., Paida, P., Popper, S., \& Brouwer, K. (2011). Under- and over-nutrition among refugees in San Diego county, California. Journal of Immigrant and Minority Health, 13(1), 161-168.

Roshania, R., Narayan, K., \& Oza-Frank, R. (2008). Age at arrival and risk of obesity among U.S. immigrants. Obesity, 16(12), 2669-2675. 
Salganik, M. J., \& Heckathorn, D. D. (2004), Sampling and estimation in hidden populations using respondent-driven sampling. Sociological Methodology, 34, 193-240. doi: 10.1111/j.0081-1750.2004.00152.x

Satia-Abouta, J., Patterson, R. E., Neuhouser, M. L., \& Elder, J. (2002). Dietary acculturation: Applications to nutrition research and dietetics. Journal of the American Dietetic Association, 102(8), 1105-1118.

Strauss, A.L., \& Corbin, J. (1990). Basics of qualitative research: Grounded theory procedures and techniques. Newbury Park, CA: Sage Publications.

Strauss, A.L. (1987). Qualitative analysis for social scientists. Cambridge, England: Cambridge University Press.

Trichopoulou, A., Soukara, S., \& Vasilopoulou, E. (2007). Traditional foods: A science and society perspective. Trends in Food Science \& Technology, 18(8), 420-427. doi:http://dx.doi.org/10.1016/j.tifs.2007.03.007

Urquhart, C. (2001). An encounter with grounded theory: Tackling the practical and philosophical issues. In E. Trauth, eds., Qualitative research in information systems: Issues and trends (pp.104-140). Hershey, PA: Idea Group Publishing;

Verbik, L., \& Lasanowski - Hobsons, V. (2007). International student mobility: Patterns and trends. New York, NY: World Education Services.

Willis, M., \& Nkwocha, O. (2006). Health and related factors for Sudanese refugees in Nebraska. Journal of Immigrant and Minority Health, 8(1), 19-33.

Winham, D. M. (2009). Culturally tailored foods and cardiovascular disease prevention. American Journal of Lifestyle Medicine, 3(1), 645-685.

Yeh, M. C., Ickes, S. B., Lowenstein, L. M., Shuval, K., Ammerman, A. S., Farris, R., \& Katz, D. L. (2008). Understanding barriers and facilitators of fruit and vegetable consumption among a diverse multi-ethnic population in the USA. Health Promotion International, 23(1), 42-51.

Zarkin, G. A., Dean, N., Mauskopf, J. A., \& Williams, R. (1993). Potential health benefits of nutrition label changes. American Journal of Public Health, 83(5), 717-724.

Appendix 1 Focus Group Questions

\section{Questions}

Dietary change 1. Tell me about what you ate before coming to the United States?

2. What foods do you eat in the United States?

a. How do you access these foods?

3. How has your diet changed since you came to the United States?

a. What caused this change if there was change?

Food access 1. How easy is it to obtain your food,

a. in terms of cost,

b. availability, and

c. preparation?

2. What is your perception of the cost of food in the United States in general? What food do you view as being "inexpensive" or "expensive"?

3. What transportation methods do you use to go to the markets where you buy your food? How often do you go? 
4. Who prepares the foods you eat here in the United States? How is this different than in your home country?

5. If you buy traditional food, where do you buy it?

Food choice 1. Describe what you eat throughout the day?

2. What influences what you eat now?

3. How do you decide on what food you eat in the United States?

4. What you feel about the quality of food compared to your home country?

5. If your diet has changed since coming to the United States, do you think it affected your health, and if so how?

\section{About the Authors}

AMIR ALAKAAM, M.B.Ch.B, M.S., is a licensed physician in Iraq and a Fulbright Scholar (2010) with a Master of Science in Nutrition from Marywood University, PA. Currently, he is a Graduate Assistant and a doctoral candidate in the Department of Nutrition and Food Systems, University of Southern Mississippi. Email: amir.alakaam@eagles.usm.edu

DiANA C. CASTEllanOS, Ph.D., R.D. is Assistant Professor in School of Education and Health Sciences, University of Dayton, OH. Email: dianacuycastellanos@gmail.com

JESSICA BODZIO, M.S., R.D., L.D.N. is a faculty and DPD director in Nutrition and Dietetics Department, Marywood University, PA. Email: jbodzio@marywood.edu

LEE HARRISON, Ph.D., R.D., F.A.D.A., C.N.S.D., C.M.F.C is a chairperson, professor in Nutrition and Dietetics Department, Marywood University, PA. Email:harrisonl@marywood.edu

$$
* * *
$$

